\title{
Management of Recurrent Breast Cancer without Systemic Therapy but with Natural Menopause as Endocrine Therapy: A Report of Two Cases
}

\author{
Hiroko Yamashita ${ }^{*}$, Tatsuya Toyama, Hiroshi Sugiura, Nobuyasu Yoshimoto, Yumi Endo, \\ Mai Iwasa, Yoshitaka Fujii and Shunzo Kobayashi
}

\author{
Oncology, Immunology and Surgery, Nagoya City University Graduate School of Medical Sciences, 1 Kawasumi, \\ Mizuho-cho, Mizuho-ku, Nagoya 467-8601, Japan
}

\begin{abstract}
We have recently managed two women of estrogen receptor (ER)-positive recurrent breast cancer without systemic therapy but with natural menopause. Case 1 was originally diagnosed with ER-positive breast cancer as a 36year-old premenopausal woman. Seven years after surgery for her primary disease, a solitary lung metastasis developed. Although she has refused endocrine therapy, natural menopause has caused regression of her lung tumor. Case 2 presented with ER-positive breast cancer as a 43-year-old premenopausal woman. Nine years after surgery, her disease relapsed with lung and pleural metastases, and she received LHRH agonist and tamoxifen as first-line treatment. After 4 years of this treatment, the disease had progressed. LHRH agonist and tamoxifen were discontinued and she has been followed without systemic therapy. She became menopausal and her metastatic disease has decreased. It is concluded that low serum estrogen levels resulting from natural menopause have acted as endocrine therapy for these patients' metastatic diseases.
\end{abstract}

Keywords: Recurrent breast cancer, premenopausal, endocrine therapy, menopause.

\section{INTRODUCTION}

Endocrine therapy is the most important systemic treatment for women with estrogen receptor (ER)-positive breast cancer. There are several approaches available to intervene in estrogen-dependent tumor growth for premenopausal women, including (1) selective ER modulators like tamoxifen that interrupt estrogen binding to its receptors and (2) estrogen deprivation strategies such as ovarian ablation or suppression [1]. Since Sir George Beatson first reported the successful treatment of young women with advanced breast cancer with bilateral oophorectomy [2], estrogen deprivation with ovarian ablation or suppression has been considered critical for premenopausal, estrogen-responsive breast cancer in both adjuvant and metastatic settings.

Recently, we have managed two cases of recurrent breast cancer in patients who refused or discontinued systemic therapy but underwent natural menopause. It appears that low serum estrogen levels due to natural menopause acted as endocrine therapy for their metastatic diseases.

\section{CASE REPORTS}

\section{Case 1}

A 36-year-old premenopausal woman with T2N0M0 stage IIA breast cancer underwent a lumpectomy and axillary dissection of her right breast at another hospital in February 1997. Pathological findings indicated that the

*Address correspondence to this author at the Oncology, Immunology and Surgery, Nagoya City University Graduate School of Medical Sciences, 1 Kawasumi, Mizuho-cho, Mizuho-ku, Nagoya 467-8601, Japan; Tel: +81-52853-8231; Fax: +81-52-853-6440; E-mail: hirokoy@med.nagoya-cu.ac.jp tumor was invasive ductal carcinoma with no lymph node metastasis, ER-positive and progesterone receptor (PgR)positive. She received tamoxifen as adjuvant therapy, but discontinued it after 1.5 years. Breast irradiation was not offered.

In March 2001, she noticed a small lump near the surgical scar of her right breast. Cytological examination revealed local recurrence of invasive ductal carcinoma. She underwent a lumpectomy followed by 50 grays of irradiation in her right breast.

In October 2003 ( 7 years after the first surgery), she visited our hospital because of an asymptomatic abnormality in a screening chest X-ray. CT and lung MRI indicated a solitary mass of $1.2 \times 1.0 \mathrm{~cm}$ in the upper lobe of her right lung, diagnosed as metastatic breast cancer. Tumor markers, such as carcinoembryonic antigen (CEA) and carbohydrate antigen (CA) 15-3, were within normal ranges.

\section{Clinical Course of Recurrent Breast Cancer (Fig. 1)}

Administration of luteinizing hormone releasing hormone (LHRH) agonist and tamoxifen was recommended for her recurrent disease, but she refused tamoxifen. After three months of LHRH agonist monotherapy, the chest X-ray revealed a decrease of the tumor in her right lung, which was evaluated as partial response. However, she stopped the therapy because of severe menopausal symptoms, such as hot flashes. Since then, we have followed her by examination of chest X-ray and serum tumor markers every three to six months, but without systemic therapy. The CT in March 2006 indicated that the metastatic tumor of her right lung had increased to $3.1 \times 2.5 \mathrm{~cm}$ without newly developed disease. The serum tumor markers and the size of the lung mass were gradually increasing, but she refused any treatment. 


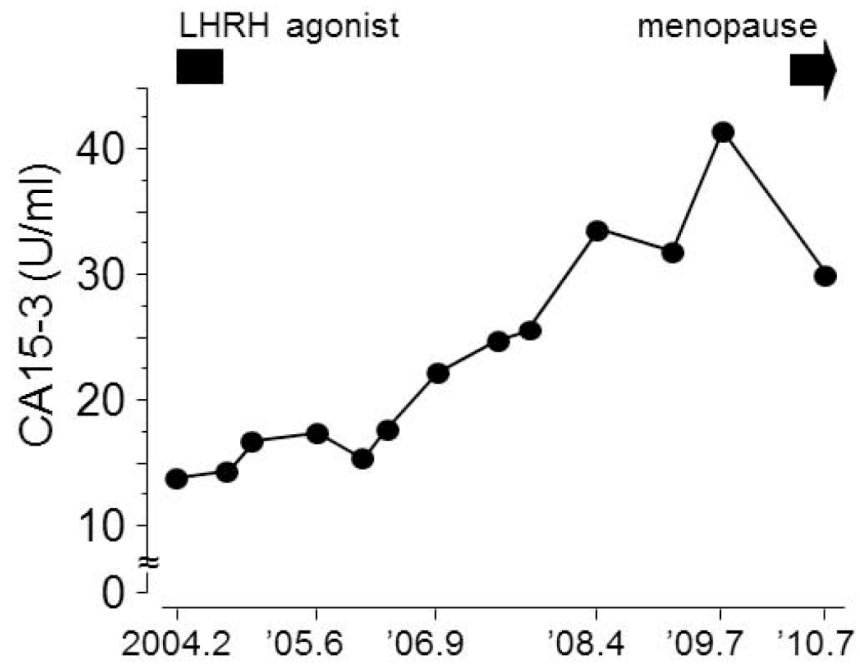

Fig. (1). Clinical course of recurrent breast cancer of Case 1. Serum CA15-3 levels were decreased after natural menopause.

Since the end of 2009 when she reached age 49, her menstruation cycle has become irregular. In January 2010, a routine chest X-ray revealed that the right lung mass had not increased compared with six months previous. In July 2010 , the tumor size was slightly decreased on X-ray, and tumor markers were also decreased. Serum estradiol levels were below $10 \mathrm{pg} / \mathrm{ml}$ and serum FSH levels were $130 \mathrm{mIU} / \mathrm{ml}$, indicating that low serum estrogen levels as a result of natural menopause have acted as endocrine therapy for her metastatic disease.

\section{Case 2}

A 43-year-old premenopausal woman with T2N1M0 stage IIB breast cancer underwent a mastectomy and axillary dissection of her right breast at another hospital in August 1996. Pathological findings indicated that the tumor was invasive ductal carcinoma, ER-positive and PgR-positive, with three lymph node metastases. She was given epirubicin $30 \mathrm{mg} /$ body for 6 cycles followed by tamoxifen for 2 years as adjuvant therapy.

In September 2005 (9 years after surgery), a screening chest X-ray indicated pleural effusion of her right lung without symptoms. CT indicated multiple masses up to the size of $1.3 \mathrm{~cm}$ in both lungs, pleural thickening, and a small volume of plural effusion in her right lung. The diagnosis was recurrent breast cancer with lung and pleural metastases. Tumor markers (CEA and CA15-3) were within normal ranges.

\section{Clinical Course of Recurrent Breast Cancer (Fig. 2)}

At age 53, still premenopausal, she was admitted to our hospital and received LHRH agonist and tamoxifen as a first-line treatment for her metastatic disease. Three months after the start of the treatment, chest X-ray revealed decreased pleural effusion in her right lung. Followup has included examination of serum tumor markers and chest Xray every three months and CT every year during stable disease.

In October 2009 (4 years after the start of the treatment for metastatic breast cancer), CT indicated that the multiple lung masses had increased in size to up to $1.6 \mathrm{~cm}$ without an increase of the right pleural effusion nor development of new lesions. Administration of LHRH agonist and tamoxifen was stopped because she was 57 years old and presumably postmenopausal. She has been followed with examination of serum tumor markers, serum estradiol and FSH levels, and chest X-rays every three months, but without systemic therapy.

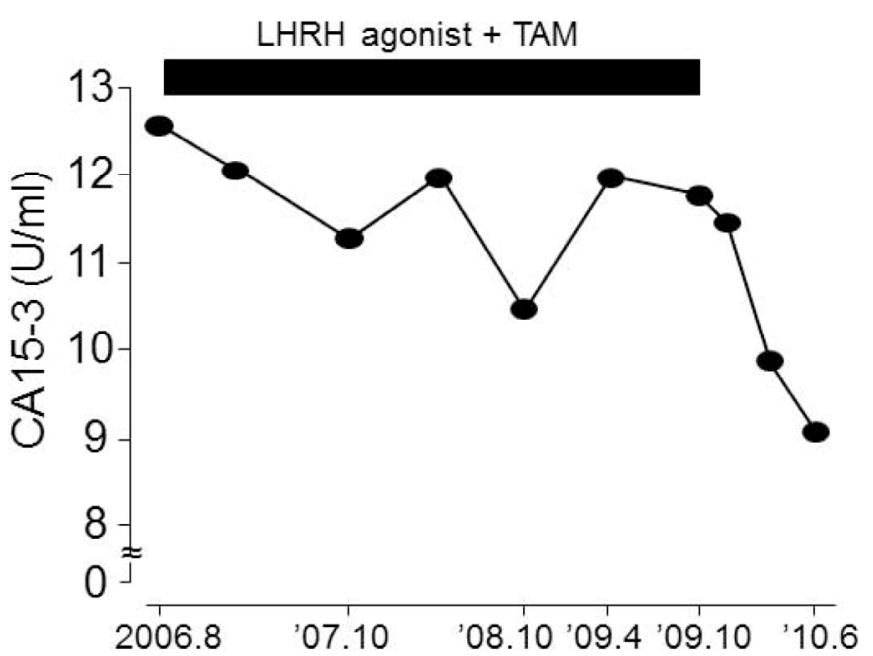

Fig. (2). Clinical course of recurrent breast cancer of Case 2. Serum CA15-3 levels were decreased after stopping administration of LHRH agonist and tamoxifen.

In February 2010 (4 months after stopping the therapy), CT indicated that the tumor sizes had not increased and there were no newly developed tumors. Tumor markers (CEA and CA15-3) were decreased in June 2010 (7 months after stopping the therapy), although both markers had been within normal ranges. Amenorrhea has continued despite cessation of LHRH agonist. Serum estradiol levels have continued below $10 \mathrm{pg} / \mathrm{ml}$ and serum FSH levels increased to $45.8 \mathrm{mIU} / \mathrm{ml}$, indicating a postmenopausal state.

\section{DISCUSSION}

In this report, we present two women of recurrent ERpositive breast cancer that have been successfully managed without systemic therapy. It is suggested that natural menopause has acted as endocrine therapy for these patients' metastatic diseases.

The combination of LHRH agonist plus tamoxifen has been considered the standard first-line endocrine therapy for premenopausal women with metastatic breast cancer [3-5]. There is no doubt that maintaining low serum estrogen levels is critical for treating premenopausal women with endocrineresponsive disease. When the combined therapy fails, LHRH agonist alone (stopping tamoxifen) might be chosen as the second-line endocrine therapy, because the long-term use of tamoxifen can generate resistance and stimulate tumor growth due to the estrogenic action of tamoxifen [6].

Case 1 refused endocrine therapy for her metastatic disease after first-line endocrine therapy with LHRH agonist alone for 3 months. Consistent with the response to the LHRH agonist, natural menopause has also been effective for her metastatic disease. Case 2 was treated with LHRH agonist and tamoxifen as first-line endocrine therapy for 4 years, then her disease progressed. Normally, tamoxifen 
would be stopped and LHRH agonist alone might be administered as the second-line treatment. However, it was not necessary for ovarian function suppression in this case, because the patient became menopausal naturally.

Both patients underwent surgery for primary breast cancer more than ten years ago, and tamoxifen for two years was standard as adjuvant endocrine therapy in ER-positive breast cancer at that time. They were free of metastatic disease for 7 years in Case 1, and 9 years in Case 2, and responded to endocrine therapy for their metastatic diseases. The St Gallen International Experts Consensus in 2009 recommended either tamoxifen or tamoxifen plus ovarian function suppression, both for the duration of 5 years, as acceptable standard treatment for premenopausal women with endocrine-responsive disease [7]. It will be important to measure serum estrogen levels during adjuvant and metastatic endocrine therapy with tamoxifen in premenopausal women, because the role of estrogen suppression, especially in the form of adjuvant endocrine therapy, has not been clearly demonstrated so far. Furthermore, several studies on the use of LHRH agonist combined with aromatase inhibitors for premenopausal breast cancer have recently been conducted in both adjuvant and metastatic settings [8-10]. Effective and adequate adjuvant endocrine therapy for premenopausal women with estrogen-responsive disease might prevent relapse.

The vast majority of metastatic breast cancer is incurable, and hence the main treatment goal is palliation, with the aim of maintaining/improving quality of life, and possibly improving survival [11]. Both patients in this report have been successfully managed without systemic therapy for some time and have maintained good quality of life, although it is not clear whether their survival has been improved. Aromatase inhibitors will be the next treatment choice if their diseases progress.

\section{ABBREVIATIONS}

$\begin{array}{ll}\mathrm{ER} & =\text { Estrogen receptor } \\ \mathrm{PgR} & =\text { Progesterone receptor } \\ \mathrm{CEA} & =\text { Carcinoembryonic antigen }\end{array}$

\section{CA15-3 = Carbohydrate antigen 15-3 \\ LHRH $=$ Luteinizing hormone releasing hormone}

\section{REFERENCES}

[1] Brown RJ, Davidson NE. Adjuvant hormonal therapy for premenopausal women with breast cancer. Semin Oncol 2006; 33: 657-63.

[2] Beatson G. On the treatment of inoperable cases of carcinoma of the mamma: suggestions for a new method of treatment with illustrative cases. Lancet 1896; 2: 104-7.

[3] Jonat W, Kaufmann M, Blamey RW, et al. A randomised study to compare the effect of the luteinising hormone releasing hormone (LHRH) analogue goserelin with or without tamoxifen in pre- and perimenopausal patients with advanced breast cancer. Eur J Cancer 1995; 31A: 137-42.

[4] Crump M, Sawka CA, DeBoer G, et al. An individual patient-based meta-analysis of tamoxifen versus ovarian ablation as first line endocrine therapy for premenopausal women with metastatic breast cancer. Breast Cancer Res Treat 1997; 44: 201-10.

[5] Klijn JG, Blamey RW, Boccardo F, et al. Combined tamoxifen and luteinizing hormone-releasing hormone (LHRH) agonist versus LHRH agonist alone in premenopausal advanced breast cancer: a meta-analysis of four randomized trials. J Clin Oncol 2001; 19: 343-53.

[6] Jordan VC. A century of deciphering the control mechanisms of sex steroid action in breast and prostate cancer: the origins of targeted therapy and chemoprevention. Cancer Res 2009; 69: 124354.

[7] Goldhirsch A, Ingle JN, Gelber RD, Coates AS, Thurlimann B, Senn HJ. Thresholds for therapies: highlights of the St Gallen International Expert Consensus on the primary therapy of early breast cancer 2009. Ann Oncol 2009; 20: 1319-29.

[8] Gnant M, Mlineritsch B, Schippinger W, et al. Endocrine therapy plus zoledronic acid in premenopausal breast cancer. N Engl J Med 2009; 360: 679-91.

[9] Forward DP, Cheung KL, Jackson L, Robertson JF. Clinical and endocrine data for goserelin plus anastrozole as second-line endocrine therapy for premenopausal advanced breast cancer. $\mathrm{Br} \mathrm{J}$ Cancer 2004; 90: 590-4.

[10] Park IH, Ro J, Lee KS, et al. Phase II parallel group study showing comparable efficacy between premenopausal metastatic breast cancer patients treated with letrozole plus goserelin and postmenopausal patients treated with letrozole alone as first-line hormone therapy. J Clin Oncol 2010; 28: 2705-11.

[11] Cardoso F, Senkus-Konefka E, Fallowfield L, Costa A, Castiglione M. Locally recurrent or metastatic breast cancer: ESMO Clinical Practice Guidelines for diagnosis, treatment and follow-up. Ann Oncol 2010; 21(5): v15-9. 